\title{
EL HABITANTE DE LA FRONTERA: RASGOS Y ROL DEL ESTRATEGA DE COMUNICACIÓN EN LAS ORGANIZACIONES
}

\author{
Ugarte Iturrizaga, Aitor \\ Madrid Salud (Ayuntamiento de Madrid) \\ ugarteia@gmail.com
}

Material original autorizado para su primera publicación en la revista académica REDMARKA. Revista Digital de Marketing Aplicado.

https://doi.org/10.17979/redma.2009.01.03.4693

\section{Aitor Ugarte Iturrizaga}

Adjunto al Gerente de Madrid Salud (Ayuntamiento de Madrid), Vicepresidente Ejecutivo para España del Foro Iberoamericano sobre Estrategias de Comunicación (FISEC) y Colaborador Honorario del Departamento de Comunicación Audiovisual y Publicidad II de la Facultad de Ciencias de la Información de la Universidad Complutense de Madrid. Su principal campo de actividad profesional y académica es la comunicación estratégica y la comunicación para la salud. Además de coordinar el curso de "Experto en Comunicación Social y Salud" de la Universidad Complutense de Madrid, es también profesor en otros cursos y títulos de posgrado en España e Iberoamérica. 


\section{Resumen:}

Se defiende en este breve ensayo la conveniencia de que el Estratega de Comunicación sea el líder de la institución en la gestión del instrumento más poderoso que tenemos los seres humanos: el lenguaje. Esa visión dota de complejidad a su tarea y la aleja de los planteamientos lineales o causa-efecto que subyacen todavía en muchas acciones marketinianas y de comunicación actuales. Este rechazo del causalismo y el logicismo clásico como principio estructurante del rol comunicativo, asume simultáneamente lo cultural, paradójico, discontinuo, contradictorio y polisémico como entorno natural de acción. Un entorno del que metafóricamente se puede afirmar que en estos momentos se parece más a una frontera que a un territorio. De ahí que se haya elegido el título de "el habitante de la frontera" para acercarse al rol y a los rasgos del Estratega de Comunicación.

Palabras clave: comunicación, estrategia, lenguaje, metáfora, frontera.

\section{Abstract: \\ THE BORDER INHABITANT: FEATURES AND ROLE OF THE COMMUNICATION STRATEGIST IN ORGANIZATIONS}

The convenience of the Communications Strategist's as the leader of the institution in the management of the most powerful tool which we human beings possess, language, is defended in this brief essay. This vision gives complexity to his work and distances it from linear or cause - effect approaches which still underlie many marketing and current communications actions. This rejection of classic cause and logic as a structural principle of the communicative role simultaneously assumes the cultural, paradoxical, discontinuous, contradictory and polysemous, as the natural environment for action. An environment of which can be metaphorically stated is more like a border than a territory in these times. From there the choice of the title "The Border Inhabitant" to approach the role and features of the Communications Strategist. 
Keywords: communication, strategy, language, metaphor, border. 
"Creo mucho en la idea de frontera como lugar de residencia."

Andrés Neuman, escritor.

"La frontera, la tierra de nadie, la no pertenencia, el territorio de la escritura."

Juan José Millás, escritor.

Las empresas, instituciones, ONGs, fundaciones, gobiernos o cualquier organización con necesidad de participar en el diálogo social asisten en su mayoría entre atónitas y átonas a la simultánea multiplicación y segmentación de sus públicos-objetivo y al aumento de las herramientas y las tecnologías mediante las que éstos se conectan y en-red-an. Nunca antes en la historia de la humanidad ha existido tanta información disponible ni ha sido tan fácil intoxicarse con ella. El problema, en el caso de la comunicación, no se plantea en términos de escasez de recursos sino de abundancia, de sobrepeso, de obesidad mórbida.

Atrás quedaron los años en que uno de los pioneros de las relaciones públicas, Edward Bernays Freud (sobrino del insigne creador del psicoanálisis), se mostraba arrepentido de haber conseguido con 20 cartas que las mujeres estadounidenses comenzaran a fumar en público (Barquero, 2002, p.41). Incluso contando con que el suceso altamente improbable existe (Taleb, 2008), ni el genial e ilustre sobrino de Sigmund Freud bastaría para que en estos días, contradictorios días, unas pocas cartas enviadas oportunamente a los receptores adecuados produjeran un efecto tan imponente como el que consiguieron aquellas "antorchas de la libertad" ${ }^{1}$ portadas por manos

\footnotetext{
${ }^{1}$ En 1929, Bernays pidió a las mujeres de la Ciudad de Nueva York que en el Desfile del Día de Pascua de aquel año, de modo provocativo, aparecieran fumando cigarrillos como una declaración de rebelión contra las normas de una sociedad dominada por los hombres. Las fotografías de lo que Bernays tituló "las antorchas de la brigada de libertad" fueron enviadas a 
femeninas. Y esto es así porque entre 1929 (año de la infeliz ocurrencia de Bernays) y 2009 poco hay en común que no sea la devoradora crisis económica que comenzó en los mercados financieros.

De 1929 a 2009: de Franklin Delano Roosevelt (al que le faltaban 4 años para llegar a la Presidencia de Estados Unidos) a Barack Houssain Obama; de John Maynard Keynes a Paul Krugman; de la aparición de Popeye y Tarzán al éxito global de los Jonas Brothers y High School Musical; del españolísimo Un Perro Andaluz de Buñuel y Dalí al anglo-estadounidense Revolutionary Road de Sam Mendes y Kate Winslet, el mundo se ha hecho más complejo y muy pocos teóricos o profesionales defienden ya que los efectos de la comunicación "de masas", por muy bien dirigida que esté, sean lineales y omnipotentes.

Sin embargo, aunque ya casi nadie porfíe en conseguir la libertad de las mujeres con 20 cartas, el hecho es que aún muchos actúan como si el paradigma no hubiera cambiado. Inconscientemente quizá, pero lo hacen. Así, algunas aproximaciones recientes a los planteamientos causa-efecto en los que creía Edward Bernays Freud han tenido y seguirán teniendo un lugar privilegiado entre los casos de estudio que ilustran los principales fracasos comunicativos de principios del S.XXI.

Uno de esos fracasos, uno de los más mencionados, se produjo el 1 de mayo de 2003 cuando el entonces presidente de los Estados Unidos, George W. Bush, a bordo del portaaviones Abraham Lincoln, dirigió un mensaje a la nación en el que no anunció el fin de las operaciones de guerra en Irak. No lo hizo pero, sin embargo, el plano que las cámaras tomaron de Bush obligaba a que la mirada de los televidentes se dirigiera hacia una enorme banderola, colocada ex profeso detrás de Bush, que soportaba con gran tipografía el lema "Misión Cumplida".

los periódicos, y muchas mujeres fueron engañadas en la creencia de que fumar era un paso importante en la lucha para la igualdad sexual. 
Muchos espectadores ante la disonancia cognitiva entre lo que estaban viendo y oyendo, pensaron de buena fe que la guerra se había acabado. Es decir, al tener que optar entre lo que percibían sus ojos y sus oídos, se quedaron inconscientemente con el mensaje visual de que la misión estaba finalizada. Se podría entender que si ésa era la intención del Gobierno de Estados Unidos, el éxito fue total. Sin embargo, más allá del logro inmediato, la tozuda realidad nos indica que seis años después de la famosa intervención de George W. Bush en el portaaviones Lincoln, las escaramuzas y los enfrentamientos (llamémosle guerra o no) en Irak continúan. Al fin y al cabo el presidente no salió bien parado de las continuas disonancias y equívocos utilizados para leer y dar forma a la realidad durante una buena parte de su mandato. De hecho, sus compatriotas fueron poco benévolos con él y le despacharon de la Presidencia con una popularidad del 30\%, algo inédito hasta la fecha.

El círculo de asesores y comunicadores de la Casa Blanca de George W. Bush erró en la estrategia. Actuó como si fuera la única fuente informativa, pero no lo era. Ya no. No tuvo en cuenta que otros muchos emisores, además del presidente de Estados Unidos, estaban trasladando simultáneamente una visión distinta -y más real- de lo que estaba sucediendo en Irak. Los ciudadanos (en Kansas y en Rosario y en Barcelona y en Hong Kong) tuvieron la oportunidad de elegir el encuadre o frame de la realidad que querían observar y finalmente secundaron la opción de quienes les trataban como interlocutores válidos no como receptores secuestrados. Dialogar siempre ha sido imposible cuando alguna de las partes pretende hacer una lectura unilateral y absoluta de la realidad.

Esta circunstancia fuerza una reflexión sobre la vigencia de los planteamientos predominantes, no solo en el marketing, la comunicación y las relaciones con los públicos, sino en la propia gestión de las organizaciones y, en definitiva, en la manera de leer la realidad y actuar sobre ella. A la cabeza de esta necesaria reflexión se hallan propuestas emergentes como la Nueva Teoría Estratégica de FISEC ${ }^{2}$ (Pérez y Massoni, 2009; Pérez, 2001), el Management 2.0 del

\footnotetext{
${ }^{2}$ Foro Iberoamericano sobre Estrategias de Comunicación.
} 
Grupo de Half Moon Bay (Hamel, 2009) o la Nueva Ilustración / The New Enligthment (Lakoff, 2008) que tanto ha tenido que ver con el advenimiento de la Era Obama.

Quienes nos dedicamos al marketing, la comunicación y a estudiar la mejor manera de relacionarnos con los públicos tenemos que asumir que el contexto ha cambiado y las prácticas que valían en tiempos de Bernays no sirven ahora, por mucho dinero e intereses que las soporten, promuevan o protejan. Vivimos una época en la que nuestro trabajo consiste, no en redactar notas, hablar con periodistas, crear eventos y asegurar una reputación corporativa consistente, sino en configurar y reconfigurar dinámicamente la trama de relaciones y de mensajes que emite y recibe nuestra institución en el diálogo que establece con sus diferentes públicos internos y externos. El diálogo y las alianzas: todo eso que le faltó a la Casa Blanca de Bush.

Digamos que el histórico jefe de prensa, profesional más bien reactivo a los requerimientos de la comunicación y poco interesado por otro público que no fueran los periodistas, va dejando paso (y lo hará aún más) a un Estratega de Comunicación que se asemeja más a la figura de un director de orquesta, que pide acompasadamente percusión al timbal y viento al clarinete, y no viceversa. Y que traslada su feedback con el lenguaje de los gestos y con la expresión.

Así tendrá que ser si aceptamos, con Paul Watzlawick y el Grupo de Palo Alto, que todos comunicamos, toda conducta tiene su vertiente comunicativa y que no se puede no comunicar (Watzlawick et al., 2006, p.49 y ss.). Aprehendidos (sí, con hache) estos axiomas, alguien se debe encargar de dar coherencia al conjunto de las comunicaciones y los diálogos que se enlazan, cruzan e incluso interrumpen en las diferentes tramas relacionales, a la búsqueda de los objetivos que establezca cada institución.

EL PODER DEL LENGUAJE Y LA IMPORTANCIA DE LA METÁFORA 
"El lenguaje está entrelazado con la vida. Lo usamos para informar y convencer, pero también para intimidar, prometer, seducir y, por supuesto, para jurar. Refleja cómo captamos la realidad, y también la imagen de nosotros mismos que intentamos proyectar hacia los demás, y los vínculos que nos atan a ellos. Se trata de una ventana a la naturaleza humana" (Pinker, 2007, p.14).

Esta cita de uno de los más afamados autores de best sellers científicos del mundo, Steven Pinker, psicólogo evolucionista y científico de la cognición en la Universidad de Harvard, nos introduce en el poder del lenguaje y de su uso relacional para crear vínculos y para cambiar la realidad social y cultural. lenguaje importa, en definitiva, porque nos permite entendernos y entender el mundo. Conectarnos. El lenguaje es la más poderosa herramienta relacional, de diálogo, con la que cuenta el Estratega de Comunicación.

$\mathrm{Y}$, en contra de los planteamientos lineales que se atienen al dualismo causaefecto, una de las características del lenguaje humano es su polisemia, el distinto significado que las personas otorgan a las mismas palabras o a los mismos gestos. Esta característica del lenguaje de los seres humanos es también lo que nos permite hablar de metáforas.

Según el planteamiento del neuro-lingüista de la Universidad de California en Berkeley, George Lakoff, "understanding language is not just nice, it is necessary. And that requires understanding the brain. The properties of the brain are what give language its power"3 (Lakoff, 2008, p.232).

Los frames o encuadres de la realidad, las narrativas propias de cada cultura, las visiones del mundo, los estereotipos, los guiones y las metáforas no solo constituyen la base de la gramática y del lenguaje, sino que son en sí mismos los elementos que configuran la forma en la que los seres humanos vemos el mundo. En las continuas comunicaciones humanas

\footnotetext{
${ }^{3}$ Traducción libre: "Entender el lenguaje no solo es agradable o bonito, es necesario. Y ello requiere entender el cerebro. Las características del cerebro son las que dan al lenguaje su poder".
} 
el cerebro y la mente -que emiten y reciben en última instancia esas comunicaciones- actúan metafóricamente. Ni universalmente ni racionalmente ni linealmente, sino metafóricamente, y en la mayoría de los casos por debajo del nivel de la conciencia.

Dentro de este universo de ideas, George Lakoff nos alerta sobre las limitaciones de la teoría de la acción racional y el mal uso que se suele hacer de ella en muchos ámbitos, entre ellos la economía y las relaciones internacionales. El autor estadounidense defiende la emergencia de una "Nueva llustración" en la que los conceptos de conciencia y racionalidad sean definidos de una manera distinta a como lo hizo la "Vieja llustración". No asumir las limitaciones de la teoría de la acción racional, sostiene Lakoff, es lo que le llevó a George W. Bush a equivocarse, entre otras cosas, con su actuación en Irak y la comunicación que de ella hizo, incluido el episodio del Abraham Lincoln. La actual crisis económica mundial, a juicio de algunos autores, tendría que ver también con la extenuación del viejo paradigma (Hamel, 2009).

No sonará nueva esta melodía a quienes hayan seguido el pensamiento de los miembros de FISEC (Foro Iberoamericano sobre Estrategias de Comunicación) que aborda desde la matriz comunicativa un nuevo paradigma para la estrategia en el que cobra especial importancia el entendimiento del hombre como un ser relacional. Frente al paradigma de la racionalidad, los miembros de FISEC ofrecemos pensar desde la "relacionalidad".

En el caso que nos ocupa, parece coherente atrapar al vuelo algunas de las ideas expuestas hasta aquí para tratar de definir el rol que puede llegar a jugar el Estratega de Comunicación, un líder de la "relacionalidad", en una organización. Por tanto, aquí van algunos de los rasgos que nos permitirán caracterizarlo:

1. Dar importancia al lenguaje,

2. Restar jerarquía a la racionalidad y al logicismo,

3. Promover lo paradójico y no-lineal, 
4. Estudiar el comportamiento del ser humano,

5. Utilizar las metáforas para hacer llegar el mensaje,

6. Introducir el factor cultural en las decisiones,

7. Saber ponerse en el lugar del otro,

8. Crear redes,

Características, actitudes y aptitudes todas ellas que, por desgracia, no suelen formar parte de las materias habituales en los lugares donde se enseña comunicación. ¿En qué Facultad las buscamos, entonces? ¿Dónde encontramos profesores, mejor maestros, que nos introduzcan en el elenco de cuestiones que son necesarias para convertirse en un Estratega de la Comunicación en el S.XXI?

La respuesta por el momento, y hasta que los estudios universitarios no abandonen el cartesianismo fragmentario que les caracteriza, habita en una metafórica frontera que justo acabamos de comenzar a explorar.

\section{EL HABITANTE DE LA FRONTERA}

Una de las primeras cuestiones que relatan aquellas personas que han volado por el espacio es que nuestro planeta, mirado desde esa enorme distancia, se percibe como diminuto y sin fronteras. Recuerdan, en definitiva, que las fronteras son convenciones acordadas o impuestas por los hombres para poder diferenciar un territorio de otro, unas competencias de otras, un departamento de otro, una facultad académica de otra, lo que es de Dios de lo que es del César. Convenciones, por cierto, dinámicas y poco predecibles estas fronteras que inventa el ser humano para parcelar la realidad y, así, se supone, tener más posibilidades de conocerla con cierta profundidad.

De la misma manera que dinámico es el uso metafórico que se le ha dado al concepto de frontera: la frontera del lejano Oeste, la Nueva Frontera de Kennedy, la frontera digital, etc. La frontera como un límite, una teórica línea de llegada que da paso a una nueva conquista, geográfica, cognitiva, política, 
tecnológica... Un ente diferenciador, que separa, que disuelve, que ansía lo objetivable.

Huyendo de este desasosegante concepto de frontera, propongo un nuevo frame, un nuevo encuadre que convierta la metáfora de la frontera en un hábitat razonable para los, por ahora, desterrados estrategas de comunicación. Un conjunto de lugares o de redes -tal vez virtuales, tal vez no- tan amplio como nuestras estrecheces lo permitan, en el que lo social y lo biológico se encuentren, y lo neurocientífico no se pelee con la filosofía, donde las emociones y la razón se hagan corpóreas, donde "los otros" sean bienvenidos al diálogo con "los nosotros", donde la estrategia se entienda como una capacidad humana y la comunicación tenga en cuenta la naturaleza relacional del hombre.

\begin{abstract}
"Ya no estamos hablando de barreras insuperables, sino de bordes permeables y mutables producidos en una dinámica, que va formando límites a los que he denominado "límites habilitantes". Estos límites no son fijos, ni rígidos, no pertenecen al universo de lo claro y distinto: son interfaces mediadoras, sistemas de intercambio y en intercambio, se caracterizan por una permeabilidad diferencial que establece una alta interconexión entre un adentro y un afuera que surge y se mantiene -o transforma- en la dinámica vincular auto-organizadora." (Najmanovich, 2008)
\end{abstract}

Quien se atreva a habitar esa frontera fluida o líquida, no tenga miedo a las paradojas, admita lo contradictorio y se considere capaz de gestionar las metáforas y los relatos de una organización para establecer relaciones con los diferentes públicos, internos y externos, es el Estratega de Comunicación que buscamos. Absténganse de presentarse al casting personas que nunca tengan dudas o que toleren mal la frustración.

CONCLUSIÓN 
El Estratega de Comunicación del S.XXI, conocedor del comportamiento humano y de las características y funciones comunicativas del lenguaje y la mente humanas, asume el rol de principal urdidor de un universo en red formado por las conexiones y vínculos de su institución con los públicos objetivo, asumiendo como eje de actuación que el cerebro humano funciona normalmente por debajo del nivel de la conciencia y que, en contra de lo que sostuvo la llustración y la ciencia que le siguió, el modo en el que la razón humana opera no es universal, lógico y apartado de lo emocional y lo corpóreo; sino todo lo contrario: inconsciente, íntimamente ligado al cuerpo, empático, emocional y metafórico.

La fragmentación derivada del cientifismo cartesiano hace poco viable en estos momentos la puesta en marcha de estudios y lugares de enseñanza donde se puedan encontrar profesores y maestros que vayan cultivando las actitudes y aptitudes necesarias para los candidatos a estrategas de comunicación. De ahí que, asumiendo la ausencia de un territorio de estudio sobre el que sustentar esta disciplina relacional, se proponga la metáfora de la frontera y de su habitante para describir el rol y los rasgos del Estratega de Comunicación. Una frontera que una y no rompa, fluida y sin muros, vinculante y no disyuntiva. Tan integradora como la vida misma que tanto se empobrece cuando las convenciones humanas convierten la política en ministerios, la educación en departamentos universitarios, el pensamiento estratégico en algo estrictamente racional y la comunicación en un fenómeno donde no se tiene en cuenta a los receptores como interlocutores válidos.

\section{BIBLIOGRAFÍA}

Barquero J.D. (2002). Comunicación y Relaciones Públicas. Madrid: Mc Graw Hill.

Hamel, G. (2009). "Moon Shots for Management". Harvard Business Review. February 2009.

Lakoff, G. (2008). The Political Mind. New York: Penguin Group.

Najmanovich, D. (2008). "La organización en redes de redes y de organizaciones". FISEC-Estrategias. Año V, Número 11, pp. 169-206. 
Pérez, R.A. y Massoni, S. (2009). Hacia una teoría general de la estrategia. Barcelona: Ariel.

Pérez, R.A. (2001). Estrategias de comunicación. Barcelona: Ariel.

Pinker, S. (2007). El mundo de las palabras. Barcelona: Paidós.

Taleb, N. (2008). El cisne negro. Barcelona: Paidós.

Watzlawick, P.; Beavin Bavelas, J. y Jackson D. (2006). Teoría de la comunicación humana. Barcelona: Herder Editorial.

\section{Para citar este articulo:}

Ugarte Iturrizaga, Aitor (21-12-2009). EL HABITANTE DE LA FRONTERA: RASGOS Y ROL DEL ESTRATEGA DE COMUNICACIÓN EN LAS ORGANIZACIONES.

REDMARKA - CIECID - Unidad de Investigación en Marketing Aplicado-Universidad de A Coruña, Número 3, V1, pp.105-117

ISSN $1852-2300$

URL del Documento : http://www.cienciared.com.ar/ra/doc.php?n=1166 